\title{
Vagoglossopharyngeal Neuralgia Treated by Microvascular Decompression and Glossopharyngeal Rhizotomy: Clinical Results of 21 Cases
}

\author{
Nan-Xiang Xiong ${ }^{\mathrm{a}}$ Hong-Yang Zhao $^{\mathrm{a}}$ Fang-Cheng Zhang $^{\mathrm{a}}$ Ru-En Liu ${ }^{\mathrm{b}}$

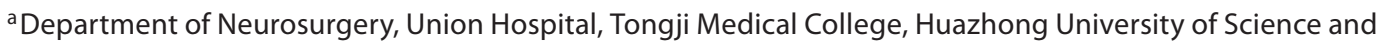 \\ Technology, Wuhan, and bepartment of Neurosurgery, China-Japan Friendship Hospital, Beijing, China
}

\section{Key Words}

Vagoglossopharyngeal neuralgia $\cdot$ Microvascular

decompression • Rhizotomy

\begin{abstract}
Background: Microvascular decompression (MVD) and rhizotomy are all selected for treating vagoglossopharyngeal neuralgia (VGPN). Nonetheless, controversies still exist about their curative effect on VGPN. Here we evaluate the effectiveness of MVD together with rhizotomy of the glossopharyngeal nerve for the treatment of VGPN. Methods: This study was carried out on 21 patients who were diagnosed with VGPN between the years 2005 and 2010. Patients underwent MVD and glossopharyngeal rhizotomy through a retromastoid keyhole approach. Surgical technique, operation results and complications were our particular concern. Results: Eighteen (85.7\%) of 21 patients experienced immediate and complete relief of pain after surgery. In the remaining 3 patients (14.3\%), the pain faded away within the following week. No patient complained of dysphonia or dysphagia. All 21 patients reported no change in their outcome at follow-up. Conclusions: Intracranial vagoglossopharyngeal nerve MVD with glossopharyngeal rhizotomy is an effective and safe procedure to treat VGPN.
\end{abstract}

Copyright $\odot 2012$ S. Karger AG, Basel

\section{KARGER}

Fax +41613061234 E-Mail karger@karger.ch www.karger.com
(C) 2012 S. Karger AG, Basel

1011-6125/12/0901-0045\$38.00/0

Accessible online at:

www.karger.com/sfn

\section{Introduction}

Vagoglossopharyngeal neuralgia (VGPN) is an uncommon craniofacial syndrome [1]. It is characterized by paroxysmal electric-shock-like pain in the innervation territory of the glossopharyngeal nerve and pharyngeal branches of the vagus nerve [2,3], usually located near the ear and gonion, as well as at the lingual base and on the pharyngeal wall [4-6]. As the concept that VGPN was caused by vascular compression like trigeminal neuralgia was fully recognized by Jannetta [7, 8] and Laha and Janetta [9], who popularized microvascular decompression (MVD) for cranial nerve neuralgias in the late 1960s, MVD has become a standard surgical treatment for cranial nerve irritative syndromes despite the fact that their underlying etiology is still incompletely understood $[8,10,11]$.

We noticed that it was reported that some VGPN cases had total pain relief without recurrence after MVD [12]. However, there were also some clinical results which showed that MVD alone did not achieve a complete healing of neurovascular compression syndrome. Treatment failure and recurrence were noted in a certain percentage of cases [8, 13-16]. VGPN was thought to be associated with sectioning of the glossopharyngeal nerve and the upper part of the vagal nerve, but, if a patient underwent upper vagal rhizotomy, it would cause a higher risk of

Tel. +86 108464 5296, E-Mail liuruen@yahoo.com.cn 
Table 1. International Headache Society diagnostic criteria for classical glossopharyngeal neuralgia

(A) Paroxysmal attacks of facial pain lasting from a fraction of a second to 2 min and fulfilling criteria B and C

(B) Pain has all of the following characteristics:

(1) unilateral location

(2) distribution within the posterior part of the tongue, tonsillar fossa, pharynx or beneath the angle of the lower jaw and/or in the ear

(3) sharp, stabbing and severe

(4) precipitated by swallowing, chewing, talking, coughing and/or yawning

(C) Attacks are stereotyped in the individual patient

(D) There is no clinically evident neurological deficit

(E) Not attributed to another disorder ${ }^{1}$

The International Headache Society has divided glossopharyngeal neuralgia into the classical and symptomatic type. In the symptomatic form, the description is as in the classical type with the proviso that aching pain may persist between paroxysms and sensory impairment may be found in the distribution of the glossopharyngeal nerve.

${ }^{1}$ Other causes have been ruled out by history, physical examination and/or special investigations.

dysphagia or vocal cord paralysis $[17,18]$. Although some scholars proposed advice on partial vagal rhizotomy [1821 , it is clinically impossible to evaluate whether the glossopharyngeal or the vagal nerve is involved at the origin of the distribution of pain, and it is also difficult to define the limit between areas innervated by the 9th and 10th cranial nerves [22]. So far, there are no standard guidelines and indications for resection of the upper vagal rootlets and decision on the number of rootlets to be sectioned.

In this article, we present a new series of 21 patients who all underwent MVD and glossopharyngeal rhizotomy for VGPN in the past 6 years. The efficiency and safety of the procedure for VGPN are discussed.

\section{Patients and Methods}

A retrospective chart review was conducted to identify the patients who had undergone MVD and rhizotomy by two surgeons between 2005 and 2010. Three patients were operated by Dr. Xiong, another 18 by Dr. Liu (only part of the patients of Dr. Liu in this period were included). Twenty-one patients suffering from medically intractable VGPN underwent MVD with glossopharyngeal rhizotomy.

\section{Patient Demographics}

Baseline clinical data are summarized as follows. The patient population in this study consisted of 8 men (38.1\%) and 13 women (61.9\%). The age range of the patients was 34-64 years (mean, 50.4 years). The majority of patients presented with left-sided symptoms $(66.7 \%, 14$ of 21 patients). On average, the patients had been symptomatic for 8.5 years before surgery (range, 3-21 years). All patients were either resistant to medical treatment or were unable to take carbamazepine because of side effects. In addition, 11 (52.3\%) of the 21 patients had unsuccessfully undergone 1 or more previous interventional therapies, including percutaneous alcohol block (6 patients), peripheral neurotomy (2 patients) and partial rhizotomy ( 3 patients). Sixteen patients were found to have hypoesthesia in the ipsilateral posterior third of the tongue or posterior pharynx. The pain disappeared transiently in 15 patients after spraying the pharynx with dicaine.

Diagnosis of typical idiopathic VGPN was confirmed if their symptoms consistently conformed to the classic description of Dandy and if they met the guidelines of the International Headache Society. Superior laryngeal neuralgia is a rare disorder characterized by severe pain in the lateral aspect of the throat, submandibular region and beneath the ear. It has been distinguished from VGPN and has not been included in the group. Diagnostic criteria are shown in table 1 [23-25].

\section{Surgical Procedure}

The patient is placed in the lateral decubitus position with the head rotated approximately $10^{\circ}$ away from the affected side and the vertex dropped $15^{\circ}$ toward the floor so that the retromastoid area is the highest point in the surgical field. All of the procedures were performed via a keyhole lateral retrosigmoid suboccipital approach. With particular care to expose the sigmoid sinus, the dura mater is opened in ' $\perp$ ' and laterally tented with tack-up sutures. Using the operating microscope, the cerebellar-pontine angle cisterns are fenestrated. After careful dissection of the arachnoid membrane around the lower cranial nerve and gentle retraction of the cerebellum by an aspirator, the root entry zone of the glossopharyngeal nerve and the upper vagal rootlets were examined. The compressing vessel, or offender, was identified near the root entry zone. Neurovascular compression was identified during the MVD procedure in all cases. Two or 4 pieces of Teflon sponge were placed between the compressing vessel and the pons to push the compressing vessel away from the glossopharyngeal nerve and upper vagal nerve. After being confirmed, the glossopharyngeal nerve was resected completely at the middle of the cisternal portion by small fine scissors.

\section{Results}

The clinical materials and operative outcome of each patient are summarized in table 2 .

\section{Intraoperative Findings}

Compressing vessels are described in detail in table 3 and can be summarized as follows: anterior inferior cerebellar artery alone in 9.5\% (2/21), posterior inferior cerebellar artery alone in $57.2 \%(12 / 21)$. A combination of 
Table 2. Summary of clinical data of 21 patients

\begin{tabular}{|c|c|c|c|c|c|c|c|}
\hline $\begin{array}{l}\text { Patient } \\
\text { No. }\end{array}$ & $\begin{array}{l}\text { Age } \\
\text { years }\end{array}$ & Sex & Side & Clinical history & Complications & Outcome & $\begin{array}{l}\text { Offending } \\
\text { vessels }\end{array}$ \\
\hline 1 & 34 & $\mathrm{~F}$ & $\mathrm{~L}$ & tongue root and ear 5 years, partial rhizotomy 2 years & dizziness & pain release immediately & PICA \\
\hline 2 & 45 & M & $\mathrm{L}$ & tonsillar fossa related to angle of jaw 7 years & tinnitus & pain release immediately & PICA \\
\hline 3 & 51 & $\mathrm{~F}$ & $\mathrm{R}$ & tonsillar fossa related to ear 10 years & none & pain release immediately & $\mathrm{PICA}+\mathrm{AICA}$ \\
\hline 4 & 58 & $\mathrm{M}$ & $\mathrm{L}$ & tongue root 13 years, percutaneous alcohol block 2 years & none & pain release immediately & PICA \\
\hline 5 & 42 & $\mathrm{~F}$ & $\mathrm{R}$ & tongue root and ear 8 years, partial rhizotomy 4 years & none & pain release immediately & AICA \\
\hline 6 & 56 & $\mathrm{~F}$ & $\mathrm{~L}$ & pharynx and ear 7 years, peripheral neurotomy 4 years & none & pain release immediately & $\mathrm{PICA}+\mathrm{VA}$ \\
\hline 7 & 61 & $\mathrm{M}$ & $\mathrm{L}$ & tongue root, tonsillar fossa 5 years & deafness & pain release immediately & AICA \\
\hline 8 & 48 & $\mathrm{~F}$ & $\mathrm{R}$ & pharynx related to mastoid process 6 years, alcohol block 3 years & none & pain release in 1 week & PICA \\
\hline 9 & 41 & M & $\mathrm{R}$ & tongue root related to gingiva 6 years & none & pain release immediately & $\mathrm{PICA}+\mathrm{VA}$ \\
\hline 10 & 57 & $\mathrm{~F}$ & $\mathrm{~L}$ & tongue root and ear 8 years, alcohol block 5 years & dizziness & pain release immediately & $\mathrm{PICA}+\mathrm{VA}$ \\
\hline 11 & 39 & $\mathrm{~F}$ & $\mathrm{~L}$ & tongue root and ear 7 years, partial rhizotomy 3 years & none & pain release in 1 week & PICA+AICA \\
\hline 12 & 54 & $\mathrm{M}$ & $\mathrm{R}$ & pharynx related to ear 5 years, peripheral neurotomy 2 years & headache & pain release immediately & PICA \\
\hline 13 & 49 & $\mathrm{~F}$ & $\mathrm{~L}$ & tongue root and pharynx 11 years & none & pain release immediately & PICA \\
\hline 14 & 61 & $\mathrm{~F}$ & $\mathrm{~L}$ & tonsillar fossa related to ear 13 years, alcohol block 7 years & facial paralysis & pain release in 1 week & $\mathrm{AICA}+\mathrm{VA}$ \\
\hline 15 & 57 & M & $\mathrm{L}$ & tongue root and angle of jaw 7 years & none & pain release immediately & PICA \\
\hline 16 & 59 & $\mathrm{M}$ & $\mathrm{R}$ & pharynx related to mastoid process and ear, 9 years & none & pain release immediately & PICA \\
\hline 17 & 64 & $\mathrm{~F}$ & $\mathrm{~L}$ & tongue root and pharynx related to gingiva, 21 years & none & pain release immediately & PICA \\
\hline 18 & 44 & $\mathrm{~F}$ & $\mathrm{R}$ & pharynx related to ear 5 years, peripheral neurotomy 4 years & none & pain release immediately & PICA \\
\hline 19 & 55 & $\mathrm{~F}$ & $\mathrm{~L}$ & pharynx related to mastoid process 8 years & dizziness & pain release immediately & PICA \\
\hline 20 & 47 & $\mathrm{M}$ & $\mathrm{L}$ & tongue root 13 years, alcohol block 3 years & none & pain release immediately & PICA+AICA \\
\hline 21 & 38 & $\mathrm{~F}$ & $\mathrm{~L}$ & tonsillar fossa related to ear 5 years & none & pain release immediately & PICA \\
\hline
\end{tabular}

PICA = Posteroinferior cerebellar artery; VA = vertebral artery; AICA = anteroinferior cerebellar artery.

multiple arterial loops was found in 7 (33.3\%) of the 21 patients. In these 7 patients, the posterior and anterior inferior cerebellar arteries were both involved in 3 cases. The posterior inferior cerebellar artery and vertebral artery were involved in 3 patients. The anterior inferior cerebellar and vertebral arteries were involved in 1 patient.

\section{Immediate Postoperative Outcomes and Follow-Up \\ Outcomes}

The symptom of VGPN disappeared immediately after surgery in 18 of 21 patients (85.7\%). In the remaining 3 patients $(14.3 \%)$, the pain slowly faded away within the following week.

All patients were followed up by telephone and/or ambulant clinic. Overall the postoperative follow-up duration was from 1 to 5 years; its mean was 3.4 years. Four patients had a follow-up duration of more than 4 years, 6 patients had one between 3 and 4 years, all patients had one of more than 1 year. All 21 patients reported complete pain relief without the need for medication, and all patients reported no change in their outcome at the follow-up.

\section{Complications}

There was no operative or perioperative mortality and no long-term surgical morbidity in this study group. No
Table 3. Offending vessel involvement

\begin{tabular}{ll}
\hline Vessel & Patients \\
\hline Single arteries & $14 / 21(66.7 \%)$ \\
PICA & $12 / 21(57.2 \%)$ \\
AICA & $2 / 21(9.5 \%)$ \\
Multiple arteries & $7 / 21(33.3 \%)$ \\
PICA + AICA & $3 / 21(14.3 \%)$ \\
AICA + VA & $1 / 21(4.8 \%)$ \\
PICA + VA & $3 / 21(14.3 \%)$ \\
PICA + AICA + VA & $0 / 21$ \\
\hline
\end{tabular}

PICA = Posteroinferior cerebellar artery; AICA = anteroinferior cerebellar artery; $\mathrm{VA}=$ vertebral artery.

wound infection and postoperative cerebrospinal fluid leak were found. Dizziness occurred in 3 and tinnitus in 1 of 21 patients, and all recovered 2 weeks later. There was 1 case of immediate postoperative mild facial weakness, and 1 case of hearing loss was observed; they all recovered completely without impairment 3 months after surgery. No patient complained of dysphonia, dysphagia or dysarthria during the perioperative period or at the long-term follow-up. By neurological examination after surgery, all 

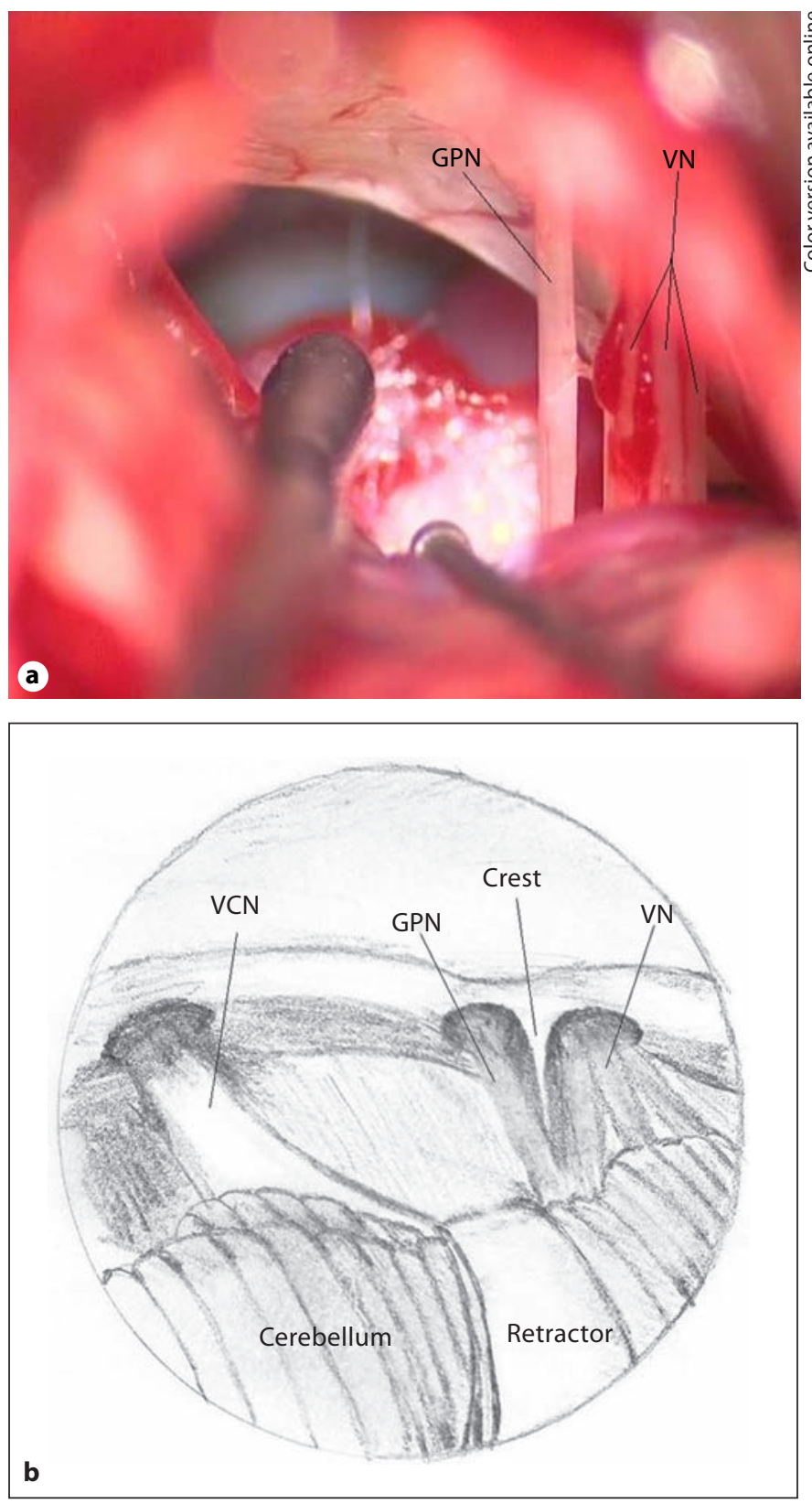

Fig. 1. Anatomical characteristics of the glossopharyngeal nerve. a Intraoperative photograph showing the details to identify the glossopharyngeal nerve (GPN). We can find that the body of the glossopharyngeal nerve is brighter than the vagus nerve (VN) under the light of the microscope, and its entrance porus is near the top of the jugular foramen. $\mathbf{b}$ Schematic drawing of the dural crest. It separates the entrance of the glossopharyngeal nerve from the vagus nerve and accessory nerve. The crest is an important anatomical landmark of the glossopharyngeal nerve. However, it could not always be seen during surgery. $\mathrm{VCN}=$ Vestibulocochlear nerve; GPN = glossopharyngeal nerve; $\mathrm{VN}=$ vagus nerve. patients were found to have hypoesthesia in the ipsilateral posterior third of the tongue and posterior pharynx and a diminished sense of taste in the ipsilateral posterior third of the tongue; 19 patients were found to have weakness of the gag reflex on the affected side. No patient complained of obvious discomfort.

\section{Discussion}

VGPN is a complex medical condition, marked by pain in the distribution of both the glossopharyngeal and vagal sensory nerve roots. Clinically, it is usually difficult to accurately specify which nerve is responsible for patient symptoms. The anatomical regions typically supplied by each nerve may vary or even overlap to a certain extent. For example, sensation of the pharynx was innervated by both the glossopharyngeal nerve and pharyngeal branch of the vagus nerve [23-26]. For greater therapeutic benefit, some scientists have suggested that both the glossopharyngeal nerve and upper rootlets (1-3) of the vagus nerve be excised during the surgical intervention for VGPN. However, the upper segment of the vagus nerve may contribute fibers to the superior or recurrent laryngeal nerve. Once severed, dysarthria and dysphagia are inevitable and permanent consequences, from which recovery is difficult. Interrupting the upper rootlets of the vagus nerve therefore carries a risk of severe complications $[18,27,29]$.

Microvascular decompression of the glossopharyngeal nerve and vagus nerve to treat VGPN was selected in some clinical centers [13-16]. The purpose of MVD is to separate the offending vessels from both nerves. Neither of the two nerves was resected in these groups but not all VGPN patients can achieve satisfactory results after MVD. Therefore, glossopharyngeal and upper vagal rhizotomies were performed in some neurosurgical centers to improve the cure rate.

However, the resection range of the nerves is still controversial. Some favor percutaneous thermal rhizotomy, others prefer extracranial section of the glossopharyngeal nerve or partial glossopharyngeal rhizotomy. However, high recurrence rates were found in these procedures after a long-term follow-up [18, 19, 25, 28, 29]. Resection of the upper vagal nerve may cause severe complications. In our surgical experience, in addition to MVD of the glossopharyngeal and vagus nerve, we did glossopharyngeal rhizotomy without upper vagal rhizotomy. By sharply dissociating the offending vessels, the ninth and tenth cranial nerves were decompressed com- 
pletely. There were no complications related to vagal nerve injury.

According to our results, all these patients had not complained of discomfort, which was relevant to glossopharyngeal rhizotomy in the follow-up period. They rarely had any subjective clinical manifestation except hypoesthesia in the ipsilateral pharynx and root of tongue. A large proportion of VGPN patients had hypoesthesia in areas innervated by the glossopharyngeal nerve, which had been found by preoperative neurological examination. So we speculated that the reason why those patients had no prominent symptoms is that they tolerated hypoesthesia in the ipsilateral pharynx and root of tongue.

Theoretically, section of the glossopharyngeal nerve may also result in paralysis of the stylopharyngeal muscle, which takes part in deglutition and vocalism. However, all patients who underwent glossopharyngeal rhizotomy had no dysarthria or dysphagia. We thought that the function of the stylopharyngeal muscle had been compensated by the anterior jugular muscle group.

The principal step in glossopharyngeal rhizotomy is to identify the glossopharyngeal nerve. Some doctors may take one rootlet of the vagus nerve for the glossopharyn- geal nerve. The proofs to identify the glossopharyngeal nerve are as below (fig. 1): (1) its entrance porus is near the top of the jugular foramen; in other words, the glossopharyngeal nerve is closest to the vestibulocochlear nerve; (2) this porus is separated from the entrance of the vagus and accessory nerves by a dural crest [30], which is an important anatomical landmark of the glossopharyngeal nerve; however, it could not always be seen in an operation; (3) the glossopharyngeal nerve body looks brighter than the vagus nerve under the light of the microscrope.

The main reason for performing glossopharyngeal nerve rhizotomy but preserving the vagus nerve is to avoid severe complications such as dysphagia or hoarseness. This procedure may be inefficient for a small proportion of vagus neuralgias. Nevertheless, the overall therapeutic effect of the procedure was satisfactory according to our clinical results. Taken together, MVD and glossopharyngeal nerve rhizotomy were a good choice for VGPN. No serious complications were found and no recurrence of pain was noted during the follow-up period. This procedure provided satisfactory results without prominent neurological complications.

\section{References}

1 Sindou M, Keravel Y: Neurosurgical treatment of vago-glossopharyngeal neuralgia. Neurochirurgie 2009;55:231-235.

-2 Evans RW, Torelli P, Manzoni GC: Glossopharyngeal neuralgia. Headache 2006;46: 1200-1202.

3 Khan NU, Iyer A: Glossopharyngeal neuralgia associated with anomalous glossopharyngeal nerve. Otolaryngol Head Neck Surg 2007; 136:502-503.

-4 Horowitz M, Horowitz M, Ochs M, Carrau R, Kassam A: Trigeminal neuralgia and glossopharyngeal neuralgia. Two orofacial pain syndromes encountered by dentists. J Am Dent Assoc 2004;135:1427-1433.

5 Soh KB: The glossopharyngeal nerve, glossopharyngeal neuralgia and the Eagle's syndrome - current concepts and management. Singapore Med J 1999;40:659-665.

-6 Ozenci M, Karaoguz R, Conkbayir C, Altin T, Kanpolat Y: Glossopharyngeal neuralgia with cardiac syncope treated by glossopharyngeal rhizotomy and microvascular decompression. Europace 2003;5:149-152.

7 Jannetta PJ: Arterial compression of the trigeminal nerve at the pons in patients with trigeminal neuralgia. J Neurosurg 2007; 107: 216-219.
8 Jannetta PJ: Observation on the aetiology of trigeminal neuralgia, hemifacial spasm, acoustic nerve dysfunction and glossopharyngeal neuralgia. Definitive microsurgical treatment and result in 117 patients. Neurochirurgia (Stutt) 1977;20:145-154.

9 Laha RK, Jannetta PJ: Glossopharyngeal neuralgia. J Neurosurg 1997;47:316-320.

-10 Ferroli P, Fioravanti A, Schiariti M, Tringali G, Franzini A, Calbucci F, Broggi G: Microvascular decompression for glossopharyngeal neuralgia: a long-term retrospectic review of the Milan-Bologna experience in 31 consecutive cases. Acta Neurochir (Wien) 2009;151:1245-1250.

11 Adams CB: Microvascular compression: an alternative view and hypothesis. J Neurosurg 1989;57:1-12.

12 Sindou M, Mertens P: Microsurgical vascular decompression (MVD) in trigeminal and glosso-vago-pharyngeal neuralgias. A twenty year experience. Acta Neurochir Suppl (Wien) 1993;58:168-170.

13 Martínez-González JM, Martínez-Rodríguez N, Calvo-Guirado JL, Brinkmann JC, Dorado CB: Glossopharyngeal neuralgia: a presentation of 14 cases. J Oral Maxillofac Surg 2011;69:38-41.
14 Kandan SR, Khan S, Jeyaretna DS, Lhatoo S, Patel NK, Coakham HB: Neuralgia of the glossopharyngeal and vagal nerves: longterm outcome following surgical treatment and literature review. Br J Neurosurg 2010; 24:441-446.

15 Fan ZM, Han YC, Li JF, Fan Z, Xu L, Wang HB: Revision surgery of the posterior fossa cranial nerves disease. Zhonghua Er Bi Yan Hou Tou Jing Wai Ke Za Zhi 2009;44:364368 .

16 Kalkanis SN, Eskandar EN, Carter BS, Barker FG 2nd: Microvascular decompression surgery in the United States, 1996 to 2000: mortality rates, morbidity rates, and the effects of hospital and surgeon volumes. Neurosurgery 2003;52:1251-1261.

- 17 Horowitz M, Horowitz M, Ochs M, Carrau $\mathrm{R}$, Kassam A: Trigeminal neuralgia and glossopharyngeal neuralgia: two orofacial pain syndromes encountered by dentists. J Am Dent Assoc 2004;135:1427-1433.

18 Taha JM, Tew JM Jr: Long-term results of surgical treatment of idiopathic neuralgias of the glossopharyngeal and vagal nerves. Neurosurgery 1995;36:926-930. 
>19 Teixeira MJ, de Siqueira SR, Bor-Seng-Shu E: Glossopharyngeal neuralgia: neurosurgical treatment and differential diagnosis. Acta Neurochir (Wien) 2008;150:471-475.

20 Owler BK, Johnston I, Kennedy M: Late recurrence of glossopharyngeal neuralgia after IXth and partial Xth nerve rhizotomy: treatment by microvascular decompression. J Neurol Neurosurg Psychiatry 2000;69:834835.

-21 Zhao K, Zuo H, Zhang L, Yu Y, Yuan Y, Guo $\mathrm{J}$ : Long-term follow-up results of microsurgical treatment for glossopharyngeal neuralgia. Zhonghua Wai Ke Za Zhi 2000;38:598600.

-22 Kondo A: Follow-up results of using microvascular decompression for treatment of glossopharyngeal neuralgia. J Neurosurg 1998;88:221-225.
23 Headache Classification Committee of the International Headache Society: Classification of diagnostic criteria for headache disorders, cranial neuralgias, and facial pain. Cephalalgia 1988;8(suppl 7):69-70.

24 Robson J, Bonica J: The vagus nerve in surgical consideration of glossopharyngeal neuralgia. J Neurosurg 1950;7:482-484.

25 Rushton J, Stevens C, Miller R: Glossopharyngeal neuralgia (vagoglossopharyngeal neuralgia): a study of 217 cases. Arch Neurol 1981;38:201-205.

26 Van Loveren HR, Tew JM Jr, Thomas G: Vago-glossopharyngeal and geniculate neuralgias in neurological surgery; in Youmans JR (ed): Neurological Surgery, ed 3. Philadelphia, Saunders, 1990, pp 3943-3949.
27 Tarlov IM: Section of the cephalic third of the vagus-spinal accessory complex: clinical and histologic results. Arch Neurol Psychiatry 1942;47:141-148.

-28 Taha JM, Tew JM Jr, Keith RW, Payner TD: Intraoperative monitoring of the vagus nerve during intracranial glossopharyngeal and upper vagal rhizotomy: technical note. Neurosurgery 1994;35:775-777.

29 Tew JM Jr: Percutaneous rhizotomy in the treatment of intractable facial pain (trigeminal, glossopharyngeal, and vagal nerves); in Schmidek HH, Sweet WH (eds): Current Techniques in Operative Neurosurgery. New York, Grune \& Stratton, 1988, pp 1111-1123.

30 Ozveren MF, Türe U, Ozek MM, Pamir MN: Anatomic landmarks of the glossopharyngeal nerve: a microsurgical anatomic study. Neurosurgery 2003;52:1400-1410. 